JOURNAL OF THE

AMERICAN MATHEMATICAL SOCIETY

Volume 21, Number 4, October 2008, Pages 1065-1084

S 0894-0347(07)00570-X

Article electronically published on April 23, 2007

\title{
THE NONCOMMUTATIVE CHOQUET BOUNDARY
}

\author{
WILLIAM ARVESON
}

\section{INTRODUCTION}

Boundary representations (defined following (1.1) below) are the noncommutative counterparts of points in the Choquet boundary of a function system in $C(X)$. The original motivation for introducing boundary representations in Arv69 was two-fold: to provide intrinsic invariants for operator systems that could be calculated for specific examples, and to provide a context for showing that the noncommutative Silov boundary exists in general. The first goal was achieved in Arv72 in which several concrete examples were worked out and applications to operator theory were developed; see Remark 1.1 for a typical example. However, the existence of boundary representations and the Šilov boundary was left open in general. Subsequently, Hamana was able to establish the existence of the noncommutative Silov boundary by making use of his theory of injective envelopes Ham79a, Ham79b. Hamana's work made no reference to boundary representations, and left untouched the question of their existence.

More recently, Muhly and Solel MS98, obtained significant results about boundary representations in an algebraic context, and Dritschel and McCullough DM05] took a major step forward by showing that every unit-preserving completely positive map of an operator system into $\mathcal{B}(H)$ can be dilated to a completely positive map with the unique extension property (see Definition 2.1). Significantly, that provided a new proof of the existence of the noncommutative Silov boundary that makes no use of injectivity. The latter authors drew motivation from previous work of Agler on a model theory for representations of non self-adjoint operator algebras (see Agl88 and references therein). On the other hand, they point out that their results seem to provide no information about the existence of boundary representations (this is discussed more fully in Remark 1.2).

An operator system is a self-adjoint linear subspace $S$ of a unital $C^{*}$-algebra that contains the unit; we usually require that the $C^{*}$-algebra be generated by $S$, and express that by writing $S \subseteq C^{*}(S)$. Let $\left\{\sigma_{x}: x \in A\right\}$ be a set of irreducible representations of $C^{*}(S)$. We say that $\left\{\sigma_{x}: x \in A\right\}$ is sufficient for $S$ if

$$
\|a\|=\sup _{x \in A}\left\|\sigma_{x}(a)\right\|, \quad a \in S
$$

Received by the editors January 12, 2007.

2000 Mathematics Subject Classification. Primary 46L07; Secondary 46L52.

Key words and phrases. Choquet boundary, operator system, completely positive maps, unique extension property.

(C)2007 American Mathematical Society Reverts to public domain 28 years from publication 
with similar formulas holding throughout the matrix hierarchy over $S$ in the sense that for every $n \geq 2$ and every $n \times n$ matrix $\left(a_{i j}\right) \in M_{n}(S)$, we have

$$
\left\|\left(a_{i j}\right)\right\|=\sup _{x \in A}\left\|\left(\sigma_{x}\left(a_{i j}\right)\right)\right\| .
$$

A boundary representation of $S$ is an irreducible representation $\pi$ of $C^{*}(S)$ on a Hilbert space whose restriction to $S$ has a unique completely positive linear extension to $C^{*}(S)$. If the set of all boundary representations for $S$ is sufficient in this sense, we say that $S$ has sufficiently many boundary representations.

In Theorem 2.2.3 of Arv69 it was shown that, in all cases in which there are sufficiently many boundary representations, the $C^{*}$-algebra $C^{*}(S)$ contains a largest closed two-sided ideal $K$ with the property that the quotient map $a \in C^{*}(S) \mapsto$ $\dot{a} \in C^{*}(S) / K$ restricts to a completely isometric map on $S$; and in such cases one has $K=\bigcap\{\operatorname{ker} \sigma: \sigma \in \partial S\}$. This ideal $K$ was called the Šilov boundary ideal for $S$ in Arv69. More recently the term has been contracted to Silov ideal, and the corresponding embedding $\dot{S} \subseteq C^{*}(S) / K$ has come to be known as the $C^{*}$-envelope of $S$. As we have already pointed out, Hamana's work implies that the Silov ideal $K$ exists in general, independently of the existence of boundary representations. Thus, the assertion that there are sufficiently many boundary representations for an operator system is equivalent in general to the assertion that the Silov ideal is the intersection of the kernels of all boundary representations. This is the proper noncommutative formulation of the statement that for every function system $S \subseteq C(X)$ that separates points of a compact Hausdorff space $X$, the closure of the Choquet boundary of $X$ (relative to $S$ ) is the Silov boundary the smallest closed subset of $X$ on which every function in $S$ achieves its norm.

Given the central role of the Choquet boundary in potential theory and other parts of commutative analysis, it is natural to expect further applications of its noncommutative generalization in the future. There has been a renewal of interest in the noncommutative Šilov boundary, beginning around 1999 with work of Blecher Ble01, and as we have already pointed out in the preceding paragraphs, those developments have been fruitful. Further results in these directions and additional references can be found in the monographs of Paulsen Pau02 and Blecher and Le Merdy BLM04.

Partly because of the promise of such developments, we were encouraged to return to the problem of the existence of boundary representations in general. In this paper we show that every separable operator system $S$ has sufficiently many boundary representations. That is accomplished by first refining the theorem of Dritschel-McCullough appropriately for separable operator systems. We then show that, given a separable Hilbert space $H$ and a UCP map $\phi: S \rightarrow \mathcal{B}(H)$ with the unique extension property, every direct integral decomposition of $\phi$ into irreducible maps gives rise to a bundle of UCP maps $\left\{\phi_{x}: x \in X\right\}$ such that $\phi_{x}$ is a boundary representation for almost every $x \in X$ with respect to the ambient measure.

The main results are Theorems 6.1, 7.1 and 8.2. There is further discussion of methodology and open problems in Section 9

Remark 1.1 (An application of boundary representations). The "intrinsic" nature of the invariants associated with boundary representations is best illustrated by an example from Arv72. If $a$ and $b$ are two irreducible compact operators with the property that the map $\lambda \mathbf{1}+\mu a \mapsto \lambda \mathbf{1}+\mu b, \lambda, \mu \in \mathbb{C}$, is completely isometric, 
then $a$ and $b$ are unitarily equivalent. Thus, an irreducible compact operator $a$ is completely determined up to unitary equivalence by the internal properties of the two-dimensional operator space

$$
S=\{\lambda \mathbf{1}+\mu a: \lambda, \mu \in \mathbb{C}\} .
$$

Indeed, in Arv72, it is shown that the identity representation of such an $S$ is a boundary representation, and that any completely isometric map of operator systems must implement a bijection of the boundary representations of one operator system to those of the other. From these results it follows that the map $\lambda \mathbf{1}+\mu a \mapsto$ $\lambda \mathbf{1}+\mu b$ extends uniquely to a $*$-homomorphism of $C^{*}$-algebras. One now deduces the above assertion from the familiar fact that an irreducible representation of the $C^{*}$-algebra of compact operators is implemented by a unitary operator (Corollary 2 of Theorem 1.4.4 of [Arv98]).

Remark 1.2 (Terminology). We caution the reader that in DM05, the term boundary representation refers rather broadly to arbitrary UCP maps with the unique extension property. In this paper we adhere to the original terminology of [Arv69] and Arv72, in which boundary representation refers to an irreducible representation of $C^{*}(S)$ whose restriction to $S$ has the unique extension property. These are the objects that generalize points of the Choquet boundary of a function system in $C(X)$ and peak points of function algebras. In particular, while the results of DM05] show that there is an abundance of maps with the unique extension property, they provide no information about the existence of boundary representations in our sense of the term.

After the first version of this paper was circulated, we learned that Marius Junge has shown in ongoing unpublished work that every subhomogeneous operator system $S \subseteq \ell^{\infty}\left(M_{n}\right), M_{n}$ denoting the algebra of $n \times n$ matrices, has sufficiently many boundary representations. While that follows from Theorem 7.1 below when $S$ is separable, Junge does not assume separability.

\section{MAXIMAL UCP MAPS OF SEPARABLE OPERATOR SYSTEMS}

In this section we discuss the unique extension property and maximality for operator-valued completely positive maps of operator systems, and we prove a refinement of a result of DM05] that will be used below.

We consider unital completely positive (UCP) maps $\phi: S \rightarrow \mathcal{B}(H)$, that is, completely positive maps that carry the unit of $S$ to the identity operator of $\mathcal{B}(H)$. Such maps satisfy $\phi\left(x^{*}\right)=\phi(x)^{*}, x \in S$. A linear map $\phi: S \rightarrow \mathcal{B}(H)$ that preserves the unit is completely positive iff it is completely contractive. We also recall that the more general theory of unital operator spaces (with unital complete contractions as maps) can be absorbed into the theory of operator systems (with UCP maps) because of the following result: If $S$ is a linear subspace of $C^{*}(S)$ containing $\mathbf{1}$, then every completely contractive unital map of $S$ extends uniquely to a UCP map of $S+S^{*}$. For these basic facts; see Propositions 1.2.8-1.2.11 of [Arv69].

As pointed out in Remark 1.2. Dritschel and McCullough have used the term boundary representation for UCP maps $\phi: S \rightarrow \mathcal{B}(H)$ that have unique completely positive extensions to representations of $C^{*}(S)$. In order to avoid conflict in terminology, in this paper we describe that property as follows: 
Definition 2.1. A UCP map $\phi: S \rightarrow \mathcal{B}(H)$ is said to have the unique extension property if

(i) $\phi$ has a unique completely positive extension $\tilde{\phi}: C^{*}(S) \rightarrow \mathcal{B}(H)$, and

(ii) $\tilde{\phi}$ is a representation of $C^{*}(S)$ on $H$.

The unique extension property for $\phi: S \rightarrow \mathcal{B}(H)$ is equivalent to the assertion that every extension of $\phi$ to a completely positive map $\phi: C^{*}(S) \rightarrow \mathcal{B}(H)$ should be multiplicative on $C^{*}(S)$. If the extension $\tilde{\phi}$ of such a map $\phi$ to $C^{*}(S)$ is an irreducible representation, then the extension is a boundary representation in the sense of [Arv69]; otherwise it is not.

Given an operator system $S \subseteq C^{*}(S)$ and two UCP maps $\phi_{k}: S \rightarrow \mathcal{B}\left(H_{k}\right)$, $k=1,2$, we write $\phi_{1} \preceq \phi_{2}$ if $H_{1} \subseteq H_{2}$ and $P_{H_{1}} \phi_{2}(x) \uparrow_{H_{1}}=\phi_{1}(x), x \in S$; in this event $\phi_{2}$ is called a dilation of $\phi_{1}$ and $\phi_{1}$ is called a compression of $\phi_{2}$. The relation $\preceq$ is transitive, and $\phi_{1} \preceq \phi_{2} \preceq \phi_{1}$ iff $H_{1}=H_{2}$ and $\phi_{1}=\phi_{2}$. Thus, $\preceq$ defines a partial ordering of UCP maps of $S$. Every UCP map $\phi: S \rightarrow \mathcal{B}(H)$ can be dilated in a trivial way by forming a direct sum $\phi \oplus \psi$ where $\psi: S \rightarrow \mathcal{B}(K)$ is another UCP map.

Definition 2.2. A UCP map $\phi: S \rightarrow \mathcal{B}(H)$ is said to be maximal if it has no nontrivial dilations: $\phi \preceq \phi^{\prime} \Longrightarrow \phi^{\prime}=\phi \oplus \psi$ for some UCP map $\psi$.

Equivalently, $\phi$ is maximal iff for every dilation $\phi^{\prime}: S \rightarrow \mathcal{B}\left(H^{\prime}\right)$ of $\phi$ acting on $H^{\prime} \supseteq H$, one has $\phi^{\prime}(S) H \subseteq H$. A dilation $\phi_{2}$ of $\phi_{1}$ need not satisfy $H_{2}=$ $\left[C^{*}\left(\phi_{2}(S)\right) H_{1}\right], C^{*}\left(\phi_{2}(S)\right)$ denoting the $C^{*}$-algebra generated by $\phi_{2}(S) \subseteq \mathcal{B}\left(H_{2}\right)$, but it can always be replaced with a smaller dilation of $\phi_{1}$ that has the property, and in that case we can assert that $\phi_{1}$ is maximal iff the only dilation $\phi_{2} \succeq \phi_{1}$ that satisfies $H_{2}=\left[C^{*}\left(\phi_{2}(S)\right) H_{1}\right]$ is $\phi_{2}=\phi_{1}$ itself. More generally, this reduction imposes an upper bound on the dimension of $H_{2}$ in terms of the dimension of $H_{1}$ and the cardinality of $S$; in particular, if $H_{1}$ is separable and $S$ is a separable operator system, then $H_{2}$ must be separable.

Remark 2.3 (Separably acting maximal dilations). A UCP map of an operator system $\phi: S \rightarrow \mathcal{B}(H)$ is said to be separably acting if $H$ is a separable Hilbert space. Let $S$ be a separable operator system and let $\phi: S \rightarrow \mathcal{B}(H)$ be a separably acting UCP map. The preceding paragraph implies that every maximal dilation $\phi^{\prime}: S \rightarrow \mathcal{B}\left(H^{\prime}\right)$ of $\phi$ can be decomposed into a direct sum of maps $\phi^{\prime}=\tilde{\phi} \oplus \lambda$ where $\tilde{\phi}$ is a separably acting maximal dilation of $\phi$ and $\lambda$ is another UCP map.

We make repeated use of the following adaptation of a result of Muhly and Solel MS98 that connects maximality to the unique extension property:

Proposition 2.4. A UCP map $\phi: S \rightarrow \mathcal{B}(H)$ has the unique extension property iff it is maximal.

Proof. Assume first that $\phi$ is maximal and let $\tilde{\phi}: C^{*}(S) \rightarrow \mathcal{B}(H)$ be a completely positive extension of it. We have to show that $\tilde{\phi}$ is multiplicative. By Stinespring's theorem, there is a representation $\sigma: C^{*}(S) \rightarrow \mathcal{B}(K)$ on a Hilbert space $K \supseteq H$ such that $\tilde{\phi}(x)=P_{H} \sigma(x) \uparrow_{H}, x \in C^{*}(S)$. We can assume that the dilation is minimal in that $K=\left[\sigma\left(C^{*}(S)\right) H\right]=\left[C^{*}(\sigma(S)) H\right]$. By maximality of $\phi, K=H$ and $\tilde{\phi}=\sigma$ is multiplicative.

Conversely, suppose that $\phi$ has the unique extension property and let $\tilde{\phi}: S \rightarrow$ $\mathcal{B}(K)$ be a dilation of $\phi$ acting on $K \supseteq H$ with $K=\left[C^{*}(\tilde{\phi}(S)) H\right]$. We show 
that $K=H$ and $\tilde{\phi}=\phi$. By Theorem 1.2.9 of Arv69 $\tilde{\phi}$ can be extended to a completely positive linear map $\psi: C^{*}(S) \rightarrow \mathcal{B}(K)$. Since the compression of $\psi$ to $H$ defines a completely positive map of $C^{*}(S)$ to $\mathcal{B}(H)$ that restricts to $\phi$ on $S$, the unique extension property implies that $P_{H} \psi P_{H}$ is multiplicative on $C^{*}(S)$. So for $x \in C^{*}(S)$,

$$
P_{H} \psi(x)^{*} P_{H} \psi(x) P_{H}=P_{H} \psi\left(x^{*} x\right) P_{H} \geq P_{H} \psi(x)^{*} \psi(x) P_{H},
$$

by the Schwarz inequality; hence $\left|\left(\mathbf{1}-P_{H}\right) \psi(x) P_{H}\right|^{2} \leq 0$. This implies that $H$ is invariant under the set of operators $\psi\left(C^{*}(S)\right) \supseteq \tilde{\phi}(S)$, and therefore under $C^{*}(\tilde{\phi}(S))$. Both $K=\left[C^{*}(\tilde{\phi}(S)) H\right]=H$ and $\tilde{\phi}=\phi$ follow.

We require the following refinement of the main result of [DM05] for separable operator systems and separably acting UCP maps:

Theorem 2.5. Let $S \subseteq C^{*}(S)$ be a separable operator system, let $H_{0}$ be a separable Hilbert space, and let $\phi_{0}: S \rightarrow \mathcal{B}\left(H_{0}\right)$ be a UCP map. Then $\phi_{0}$ can be dilated to a separably acting UCP map $\phi: S \rightarrow \mathcal{B}(H)$ with the unique extension property.

Remark 2.6 (Coherent sequences of UCP maps). In the proof of Theorem 2.5 we make repeated use of the following observation. Let $H_{1} \subseteq H_{2} \subseteq \cdots$ be an increasing sequence of subspaces of a Hilbert space $H=\overline{\bigcup_{n} H_{n}}$. Given a sequence of UCP maps $\phi_{n}: S \rightarrow \mathcal{B}\left(H_{n}\right)$ that is coherent in the sense that $\phi_{n} \preceq \phi_{n+1}$ for $n \geq 1$, there is a unique UCP map $\phi: S \rightarrow \mathcal{B}(H)$ that satisfies $\phi_{n} \preceq \phi$ for all $n$. The proof is a straightforward exercise based on the following elementary fact: For every sequence of operators $a_{n} \in \mathcal{B}\left(H_{n}\right)$ such that $\sup _{n}\left\|a_{n}\right\|<\infty$ and $a_{n}=P_{H_{n}} a_{n+1}\left\lceil_{H_{n}}, n \geq 1\right.$, there is a unique operator $a \in \mathcal{B}(H)$ satisfying $P_{H_{n}} a \uparrow_{H_{n}}=a_{n}, n=1,2, \ldots$ Moreover, one obviously has $\|a\|=\sup _{n}\left\|a_{n}\right\|$.

Let $\phi: S \rightarrow \mathcal{B}(H)$ be a UCP map and let $F$ be a subset of $S \times H$. We will say that $\phi$ is maximal on $F$ if for every dilation $\psi$ of $\phi$ acting on $K \supseteq H$, we have

$$
\psi(x) \xi=\phi(x) \xi, \quad(x, \xi) \in F .
$$

A UCP map $\phi: S \rightarrow \mathcal{B}(H)$ is maximal iff it is maximal on $S \times H$. Note that if $\phi$ is maximal on $F \subseteq S \times H$ and $\psi \succeq \phi$, then $\psi$ is maximal on $F$ as well. Our proof of Theorem 2.5 uses the following result, inspired by an observation of N. Ozawa.

Lemma 2.7. Let $S$ be a separable operator system. For every UCP map $\phi: S \rightarrow$ $\mathcal{B}(H)$ where $H$ is a separable Hilbert space and every $(x, \xi) \in S \times H$, there is a separably-acting dilation of $\phi$ that is maximal on $(x, \xi)$.

Proof. Since for every dilation $\psi \succeq \phi$ we have $\|\psi(x) \xi\| \leq\|x\| \cdot\|\xi\|<\infty$, we can find a separably acting dilation $\phi_{1}$ of $\phi$ for which $\left\|\phi_{1}(x) \xi\right\|$ is as close to $\sup \{\|\psi(x) \xi\|$ : $\psi \succeq \phi\}$ as we wish. Continuing inductively, we find a sequence of separably acting UCP maps $\phi \preceq \phi_{1} \preceq \phi_{2} \preceq \cdots$ such that $\phi_{n}: S \rightarrow \mathcal{B}\left(H_{n}\right), H \subseteq H_{1} \subseteq H_{2} \subseteq \cdots$, and

$$
\left\|\phi_{n+1}(x) \xi\right\| \geq \sup _{\psi \succeq \phi_{n}}\|\psi(x) \xi\|-1 / n .
$$

Let $H_{\infty}$ be the closure of the union $\bigcup_{n} H_{n}$ and let $\phi_{\infty}: S \rightarrow \mathcal{B}\left(H_{\infty}\right)$ be the unique UCP map that compresses to $\phi_{n}$ on $H_{n}$ for every $n$ (see Remark 2.6). Note that $\phi_{\infty}$ is maximal on $(x, \xi)$. Indeed, if $\psi \succeq \phi_{\infty}$, then $\psi \succeq \phi_{n}$ for every $n \geq 1$. Fixing $n \geq 1$ and noting that $\xi \in H_{n+1}$, we have

$$
\left\|\phi_{\infty}(x) \xi\right\| \geq\left\|P_{H_{m+1}} \phi_{\infty}(x) \xi\right\|=\left\|\phi_{n+1}(x) \xi\right\| \geq\|\psi(x) \xi\|-1 / n
$$


so that $\left\|\phi_{\infty}(x) \xi\right\| \geq\|\psi(x) \xi\|$ because $n$ can be arbitrarily large. Hence

$$
\left\|\psi(x) \xi-\phi_{\infty}(x) \xi\right\|^{2}=\left\|\psi(x) \xi-P_{H_{\infty}} \psi(x) \xi\right\|^{2}=\|\psi(x) \xi\|^{2}-\left\|\phi_{\infty}(x) \xi\right\|^{2} \leq 0
$$

so that $\psi(x) \xi=\phi_{\infty}(x) \xi$. The assertion follows since $\overline{\bigcup_{n} H_{n+1}}=H_{\infty}$.

Proof of Theorem 2.5. We claim first that $\phi_{0}$ can be dilated to a separably acting UCP map $\phi_{1}: S \rightarrow \mathcal{B}\left(H_{1}\right)$ that is maximal on $S \times H_{0}$. To that end, let $C$ be a countable dense subset of $S$, let $D$ be a countable norm-dense subset of $H_{0}$, and enumerate the elements of $C \times D=\left\{z_{1}, z_{2}, \ldots\right\}$. We claim that there is a sequence of separably acting UCP maps $\omega_{n}: S \rightarrow \mathcal{B}\left(K_{n}\right), n \geq 1$, such that

(i) $\phi_{0} \preceq \omega_{1} \preceq \omega_{2} \preceq \cdots$, and

(ii) $\omega_{n}$ is maximal on $\left\{z_{1}, \ldots, z_{n}\right\}$.

Indeed, Lemma 2.7 implies the existence of a separably acting dilation $\omega_{1}$ of $\phi_{0}$ that is maximal on $z_{1}$. Given that $\omega_{1}, \ldots, \omega_{n}$ have been defined and satisfy (i) and (ii), the same reasoning gives a separably acting dilation $\omega_{n+1}$ of $\omega_{n}$ that is maximal on $z_{n+1}$; since $\omega_{n+1}$ dilates each of the preceding maps, it must also be maximal on $z_{1}, \ldots, z_{n}$. Once one is given such a sequence $\omega_{1}, \omega_{2}, \ldots$, one can let $H_{1}$ be the closure of $\bigcup_{n} K_{n}$ and let $\phi_{1}$ be the unique UCP map of $S$ into $\mathcal{B}\left(H_{1}\right)$ that compresses to $\omega_{n}$ on each $K_{n}$.

By an obvious induction on the preceding fact, one obtains an increasing sequence of separable Hilbert spaces $H_{0} \subseteq H_{1} \subseteq H_{2} \subseteq \cdots$ and UCP maps $\phi_{n}: S \rightarrow \mathcal{B}\left(H_{n}\right)$ such that $\phi_{n+1}$ is a dilation of $\phi_{n}$ that is maximal on $S \times H_{n}, n=0,1,2, \ldots$. Let $H_{\infty}$ be the closure of $\bigcup_{n} H_{n}$ and let $\phi_{\infty}: S \rightarrow \mathcal{B}\left(H_{\infty}\right)$ be the unique UCP map that compresses to $\phi_{n}$ on $H_{n}$ for every $n \geq 1$. Note that for every dilation $\psi: S \rightarrow \mathcal{B}(K)$ of $\phi_{\infty}$ and every $n \geq 1$, both $\psi$ and $\phi_{\infty}$ are dilations of $\phi_{n+1}$, so by maximality of $\phi_{n+1}$ on $S \times H_{n}$ we have

$$
\psi(x) \xi=\phi_{n+1}(x) \xi=\phi_{\infty}(x) \xi, \quad(x, \xi) \in S \times H_{n} .
$$

It follows that $\phi_{\infty}$ is maximal on $S \times \bigcup_{n} H_{n}$, hence on its closure $S \times H_{\infty}$.

Remark 2.8 (Significance of the relation $\phi \preceq \psi$ ). There is a weaker and considerably more flexible ordering $\prec$ of UCP maps, in which for UCP maps $\phi_{k}: S \rightarrow \mathcal{B}\left(H_{k}\right)$, $k=1,2, \phi_{1} \prec \phi_{2}$ means that there is an isometry $V: H_{1} \rightarrow H_{2}$ such that $\phi_{1}(a)=V^{*} \phi_{2}(a) V, a \in S$. Equivalently, $\phi_{1}$ is unitarily equivalent to a map $\phi_{1}^{\prime}$ satisfying $\phi_{1}^{\prime} \preceq \phi_{2}$. While there is a variation of Theorem 2.5 that makes a similar assertion about the maximality properties of the relation $\prec$, one cannot prove it by a verbatim replacement of $\preceq$ with $\prec$ in the above arguments. This subtle difficulty becomes apparent when one attempts to associate a single "limit" UCP map with a weakly increasing sequence $\phi_{1} \prec \phi_{2} \prec \cdots$ as we did in Remark 2.6.

\section{Borel CROSS SECTIONS}

Given standard Borel spaces $X, Y$ and a surjective Borel map $f: X \rightarrow Y$, a cross section for $f$ is a Borel function $g: Y \rightarrow X$ such that $f \circ g$ is the identity map of $Y$. Not every surjective Borel map of standard Borel spaces has a Borel cross section. Indeed, there exist Borel subsets $X$ of the unit square $[0,1] \times[0,1]$ with the property that the projection $p(x, y)=x$ maps $X$ onto $[0,1]$, but $p$ does not have a Borel cross section [Lus30, [Nov31; a simpler example was given by Blackwell in Bla68. The following result provides the measure-theoretic substitute that we require. 
Theorem 3.1. Let $X, Y$ be standard Borel spaces, let $f: X \rightarrow Y$ be a surjective Borel map, and let $\mu$ be a finite positive measure on $Y$. Then there is a Borel set $N \subseteq Y$ of measure zero and a Borel map $g: Y \backslash N \rightarrow X$ such that $f \circ g$ is the identity map on $Y \backslash N$.

Versions of Theorem 3.1 are part of the lore of the subject that originates in work of von Neumann, and later, Mackey. But since we lack a convenient reference and the result is needed below, we briefly indicate how one deduces it from a selection theorem proved in Arv98. Recall that a subset $A \subseteq X$ of a standard Borel space $X$ is said to be absolutely measurable if, for every finite positive measure $\mu$ on $X$, there are Borel sets $E_{\mu}, F_{\mu}$ such that $E_{\mu} \subseteq A \subseteq F_{\mu}$ and $\mu\left(F_{\mu} \backslash E_{\mu}\right)=0$. The class of all absolutely measurable subsets of $X$ is a $\sigma$-algebra containing the Borel sets. Analytic sets are examples of absolutely measurable sets that need not be Borel sets (see Section 3.4 of [Arv98]). A function $f: X \rightarrow Y$ from a standard Borel space $X$ to a Borel space $Y$ is said to be absolutely measurable if $f^{-1}(E)$ is absolutely measurable for every Borel set $E \subseteq Y$. Theorem 3.4.3 of Arv98 specializes to the following assertion in this context:

Theorem 3.2. Let $X$ be a standard Borel space and let $Y$ be a countably separated Borel space. Then every surjective Borel map $f: X \rightarrow Y$ has an absolutely measurable cross section.

To deduce Theorem 3.1 from Theorem 3.2, note that for every finite measure $\mu$ on $Y$, an absolutely measurable cross section $g: Y \rightarrow X$ for $f$ must agree almost everywhere $(d \mu)$ with a Borel function $g_{\mu}: Y \rightarrow X$, where of course $g_{\mu}$ depends on $\mu$. Indeed, this is obvious if $X$ is finite or countable; if $X$ is uncountable, then it is Borel isomorphic to the unit interval $[0,1], g$ becomes a real valued function in $L^{\infty}(Y, \mu)$, and such a function must agree with a Borel function $g_{\mu}$ almost everywhere $(d \mu)$. Letting $N \subseteq Y$ be a Borel set of $\mu$-measure zero such that $g=g_{\mu}$ on $Y \backslash N$, one obtains $f \circ g_{\mu}(y)=f \circ g(y)=y$ for all $y \in Y \backslash N$, and Theorem 3.1 follows.

We also require the following result, which follows from Theorem 3.3.4 of [Arv98]. Recall that a subset $A$ of a standard Borel space $X$ is said to be analytic if there is an analytic set in a Polish space that is isomorphic to $A$ with its relative Borel structure.

Theorem 3.3. Let $X, Y$ be standard Borel spaces and let $f: X \rightarrow Y$ be a Borel map. Then $f(X)$ is an analytic set in $Y$ and is therefore absolutely measurable.

\section{Borel families of UCP MAPS}

In this section we discuss families of UCP maps of $S$ and their basic measurability properties. Throughout, $S$ will denote a separable operator system and $X$ will denote a standard Borel space.

Operator algebraists traditionally refer to Chapitre II of [Dix57] for the basic disintegration theory of representations of $C^{*}$-algebras. We prefer a different though roughly equivalent - formulation that is based on standard Borel spaces, and which runs more parallel to the modern theory of vector bundles over topological spaces. Thus we shall formulate the basic structures with some care, so that key results in the following sections concerning decomposable maps of operator spaces can be readily deduced from the development of Arv98, Chapters 3 and 4 . For clarity, we have included more generality (and more detail) than is actually required below. 
Let $H=\left\{H_{x}: x \in X\right\}$ be a standard bundle of separable Hilbert spaces over $X$. More precisely, we are given a standard Borel space $H$ and a surjective Borel map $p: H \rightarrow X$ with the property that $H_{x}=p^{-1}(x)$ is a separable Hilbert space for every $x$, such that vector addition, multiplication by scalars, and the inner product are Borel-measurable. Notice that both vector addition and the inner product are defined on the following Borel subset of $H \times H$ :

$$
B=\{(\xi, \eta) \in H \times H: p(\xi)=p(\eta)\} \subseteq H \times H,
$$

and the requirements are that both functions $(\xi, \eta) \in B \mapsto \xi+\eta \in H$ and $(\xi, \eta) \in$ $B \mapsto\langle\xi, \eta\rangle \in \mathbb{C}$ should be Borel-measurable. Measurability of scalar multiplication means that the function

$$
(\lambda, \xi) \in \mathbb{C} \times H \mapsto \lambda \cdot \xi \in H
$$

should be Borel. If one is given a complex-valued Borel function $\mu: H \rightarrow \mathbb{C}$, then the function $\xi \in H \mapsto \mu(\xi) \xi \in H$ can be expressed as the composition of the Borel function $\xi \in E \mapsto(\mu(\xi), \xi) \in \mathbb{C} \times E$, with the Borel function $(\lambda, \xi) \in$ $\mathbb{C} \times E \mapsto \lambda \xi \in E$, and is therefore a Borel function. It follows from this observation that for every complex-valued Borel function $\lambda: X \rightarrow \mathbb{C}$ and every Borel section $x \in X \mapsto \xi(x) \in H_{x}$ of $p: H \rightarrow X$, the function

$$
x \in X \mapsto \lambda(x) \xi(x)=\lambda(p(\xi(x))) \xi(x)=\lambda \circ p(\xi(x)) \xi(x)
$$

is another Borel section; indeed, it is the composition of the Borel function $x \mapsto \xi(x)$ with the above map $\xi \mapsto \mu(\xi) \xi$ in which $\mu=\lambda \circ p$.

Though it is not necessary for the development, it will be convenient to assume that $H_{x} \neq\{0\}$ for every $x \in X$.

The last and most important axiom for a standard Hilbert bundle is that there should exist a sequence of Borel sections $\xi_{n}: x \in X \mapsto \xi_{n}(x) \in H_{x}, n=1,2, \ldots$, such that $H_{x}$ is the closed linear span of $\left\{\xi_{1}(x), \xi_{2}(x), \ldots\right\}$ for every $x \in X$. There is a natural notion of (unitary) isomorphism of Hilbert bundles $p^{k}: H^{k} \rightarrow X$ over $X$, $k=1,2$, namely an isomorphism of Borel spaces $U: H^{1} \rightarrow H^{2}$ with the property that $U$ restricts to a unitary operator from $H_{x}^{1}$ to $H_{x}^{2}$ for every $x \in X$.

For each $n=\infty, 1,2, \ldots$, let $X_{n}=\left\{x \in X: \operatorname{dim} H_{x}=n\right\}$. Though it is formulated in a somewhat different setting, the proof of Lemme 1 on p. 139 of Dix57 shows that $X_{n}$ is a Borel set for every $n$. Moreover, for finite $n$, the Gram-Schmodt procedure utilized in its proof generates a sequence of Borel sections $x \in X_{n} \mapsto e_{k}(x) \in H_{x}, 1 \leq k \leq n$, such that $\left\{e_{1}(x), \ldots, e_{n}(x)\right\}$ is an orthonormal basis for $H_{x}$ for every $x \in X_{n}$. If $n=\infty$, we similarly obtain a Borel-measurable orthonormal basis $\left\{e_{1}(x), e_{2}(x), \ldots\right\}$ for $H_{x}$ for every $x \in X_{\infty}$.

It follows that the restriction of the bundle to $X_{n}$ is isomorphic to the trivial bundle $p: X_{n} \times \mathbb{C}^{n} \rightarrow X_{n}$ if $n<\infty$, or $p: X_{\infty} \times \ell^{2} \rightarrow X_{\infty}$ if $n=\infty$. For example, for finite $n$ one can define a unitary operator $U_{x}: H_{x} \rightarrow \mathbb{C}^{n}$ for every $x \in X_{n}$ by

$$
U_{x}(\xi)=\left(\left\langle\xi, e_{1}(x)\right\rangle, \ldots,\left\langle\xi, e_{n}(x)\right\rangle\right) \in \mathbb{C}^{n}, \quad \xi \in H_{x},
$$

and one obtains a trivializing isomorphism $U: H \uparrow_{X_{n}} \rightarrow X_{n} \times \mathbb{C}^{n}$ as the total map $U(x, \xi)=\left(x, U_{x} \xi\right)$ associated with this family of unitary operators.

As an illustration, using the above remarks it is not hard to show that a section $x \in X \mapsto \eta(x) \in H_{x}$ is Borel-measurable iff each of the complex-valued inner products $x \in X \mapsto\left\langle\eta(x), \xi_{n}(x)\right\rangle, n=1,2, \ldots$, is a Borel function (see Remark 4.2 for more details). More generally, these remarks show that one can reduce the analysis of standard Hilbert bundles to the case of flat Hilbert bundles, and this procedure of 
"flattening and piecing together" is useful for clarifying the nature of more complex structures associated with a Hilbert bundle, as we will see momentarily.

There is also a natural notion of a bounded measurable family of operators $A=$ $\left\{A_{x}: x \in X\right\}$ on a Hilbert bundle, namely a Borel map $A: H \rightarrow H$ that restricts to a bounded linear operator $A_{x} \in \mathcal{B}\left(H_{x}\right)$ on each fiber such that $\sup _{x \in X}\left\|A_{x}\right\|<\infty$. The set of all such operator families is a unital $C^{*}$-algebra. More generally, there is a natural notion of a weak measurability for a family $\phi=\left\{\phi_{x}: x \in X\right\}$ of UCP maps of $S$ :

Definition 4.1. By a family of UCP maps on $S$ we mean a set $\left\{\phi_{x}: x \in X\right\}$ of UCP maps $\phi_{x}: S \rightarrow \mathcal{B}\left(H_{x}\right)$ indexed by $X$ with the property that for every pair of Borel sections $\xi, \eta$ of $H$ and for every $s \in S$, the inner product $x \mapsto\left\langle\phi_{x}(s) \xi(x), \eta(x)\right\rangle$ defines a complex-valued Borel function on $X$.

We will write a family of UCP maps in the more descriptive way as

$$
\phi=\left\{\phi_{x}: S \rightarrow \mathcal{B}\left(H_{x}\right): x \in X\right\} .
$$

A family of UCP maps is a Borel cross section of a bundle of UCP maps that is described as follows. For each $x \in X$, the set $\operatorname{UCP}\left(S, \mathcal{B}\left(H_{x}\right)\right)$ of all UCP maps from $S$ into $\mathcal{B}\left(H_{x}\right)$ is a convex set of linear maps defined on $S$. The total space of this collection of maps is

$$
U C P(X, S, \mathcal{B}(H))=\left\{(x, \phi): x \in X, \quad \phi \in U C P\left(S, \mathcal{B}\left(H_{x}\right)\right\},\right.
$$

with projection $p: U C P(X, S, \mathcal{B}(H)) \rightarrow X$ given by $p(x, \phi)=x$, and one has $U C P\left(S, \mathcal{B}\left(H_{x}\right)\right)=p^{-1}(x)$.

There is a natural way to make $U C P(X, S, \mathcal{B}(H))$ into a standard Borel space in such a way that $p$ is a Borel map. To see that, assume first that the fibers $H_{x}$ do not depend on $x$, say $H_{x}=K, x \in X, K$ being a separable Hilbert space. Then for each $x \in X$ we have

$$
U C P\left(S, \mathcal{B}\left(H_{x}\right)\right)=U C P(S, \mathcal{B}(K))
$$

and therefore $p: U C P(X, S, \mathcal{B}(H)) \rightarrow X$ becomes the trivial family of sets

$$
p: X \times U C P(S, \mathcal{B}(K)) \rightarrow X .
$$

The space of maps $U C P(S, \mathcal{B}(K))$ carries a BW-topology (see Arv69), and since $S$ and $K$ are both separable, this topological space is metrizable and compact. Hence the cartesian product $X \times U C P(S, \mathcal{B}(K))$ is a standard Borel space and $p$ becomes a Borel map for which $p^{-1}(x)=U C P(S, \mathcal{B}(K))$. So in this case, $U C P(X, S, \mathcal{B}(H))$ is a trivial standard Borel bundle of compact convex metrizable spaces of linear maps $p: X \times U C P(S, K) \rightarrow X$.

If $p: H \rightarrow X$ is merely isomorphic to a trivial bundle $p: X \times K \rightarrow X$ of separable Hilbert spaces, then one can make use of that isomorphism of Hilbert bundles in the obvious way to transfer the Borel structure of the flat bundle $p$ : $X \times U C P(S, \mathcal{B}(K)) \rightarrow X$ to $p: U C P(X, S, \mathcal{B}(H)) \rightarrow X$. Finally, in the general case one decomposes $X=X_{\infty} \cup X_{1} \cup X_{2} \cup \cdots$ into a disjoint union, thereby decomposing the space of maps $U C P(X, S, \mathcal{B}(H))$ into a disjoint union, and one introduces a (standard) Borel structure on this disjoint union of standard Borel spaces in the usual way. Thus, this "flattening and piecing together" procedure allows one to introduce a standard Borel structure on the bundle of maps $p$ : $U C P(X, S, \mathcal{B}(H)) \rightarrow X$. 
Remark 4.2 (Measurability of sections). Let $\left\{H_{x}: x \in X\right\}$ be a standard Hilbert bundle and suppose that, for each $x \in X$, we have a UCP map $\phi_{x}: S \rightarrow \mathcal{B}\left(H_{x}\right)$. The function $x \in X \mapsto \phi_{x} \in U C P\left(S, \mathcal{B}\left(H_{x}\right)\right)$ is a section of the bundle of maps

$$
p: U C P(X, S, \mathcal{B}(H)) \rightarrow X,
$$

and Definition 4.1 makes an assertion about the measurability of this section that is not identical verbatim with the definition of measurability that accompanies the Borel structure defined on $U C P(X, S, \mathcal{B}(H))$. Since this is a central issue, we sketch a proof that the two definitions of measurability are in fact equivalent.

Indeed, according to the "flattening and piecing together" procedure, it suffices to discuss measurability of sections of a trivial bundle of maps

$$
p: X \times U C P(S, \mathcal{B}(K)) \rightarrow X,
$$

where $K$ is a separable Hilbert space. The Borel structure on this bundle is the product Borel structure, where the Borel structure of $\operatorname{UCP}(S, \mathcal{B}(K))$ is generated by its relative BW-topology - namely the weakest topology on $U C P(S, \mathcal{B}(K))$ that makes all of the following functions continuous:

$$
\phi \in U C P(S, \mathcal{B}(H)) \mapsto\langle\phi(s) \xi, \eta\rangle \in \mathbb{C},
$$

where $s$ ranges over $S$ and $\xi, \eta$ range over $K$; note that one can restrict $s$ to the set of self-adjoint elements of $S$ because $\phi$ preserves adjoints. After this description of the BW-topology, it follows that a section

$$
x \in X \mapsto \phi_{x} \in U C P(S, \mathcal{B}(K))
$$

is Borel measurable iff $x \mapsto\left\langle\phi_{x}(s) \xi, \eta\right\rangle$ is a complex-valued Borel function for every $s=s^{*} \in S$ and every $\xi, \eta \in K$.

Definition 4.1 differs from this in that it requires $x \mapsto\left\langle\phi_{x}(s) \xi(x), \eta(x)\right\rangle$ to be Borel measurable for every $s=s^{*}$, and every pair of Borel functions $x \mapsto \xi(x) \in K$, $x \mapsto \eta(x) \in K$. Thus, the equivalence of the two notions of measurability follows from the equivalence of the following assertions about self-adjoint operator functions $x \in X \mapsto A_{x}=A_{x}^{*} \in \mathcal{B}(K)$ :

(i) For each $\xi \in K$, the function $x \in X \mapsto A_{x} \xi \in K$ is Borel measurable.

(ii) For each $\xi, \eta \in K$, the complex-valued function $x \in X \mapsto\left\langle A_{x} \xi, \eta\right\rangle$ is Borel measurable.

(iii) For every pair of Borel functions $\xi, \eta: X \rightarrow K$, the complex-valued function $x \mapsto\left\langle A_{x} \xi(x), \eta(x)\right\rangle$ is Borel measurable.

Indeed, the equivalence of (i) and (ii) follows from the fact that $K$ is a Polish space relative to its norm topology, and that the sigma algebra generated by the weak topology of $K$ coincides with the sigma algebra generated by its norm topology (for example, see Theorem 3.3.5 of [Arv98]). Obviously (iii) $\Longrightarrow$ (ii), and (ii) $\Longrightarrow$ (iii) becomes obvious as well after one expands the inner product of (iii) using an orthonormal basis $e_{1}, e_{2}, \ldots$ for $K$,

$$
\begin{aligned}
\left\langle A_{x} \xi(x), \eta(x)\right\rangle & =\sum_{n}\left\langle A_{x} \xi(x), e_{n}\right\rangle\left\langle e_{n}, \eta(x)\right\rangle=\sum_{n}\left\langle\xi(x), A_{x} e_{n}\right\rangle\left\langle e_{n}, \eta(x)\right\rangle \\
& =\sum_{m, n}\left\langle\xi(x), e_{m}\right\rangle\left\langle e_{m}, A_{x} e_{n}\right\rangle\left\langle e_{n}, \eta(x)\right\rangle .
\end{aligned}
$$




\section{Sections of the Dilation Bundle}

Throughout this section, $\left\{H_{x}: x \in X\right\}$ will denote a standard Borel Hilbert bundle and $\phi=\left\{\phi_{x}: S \rightarrow \mathcal{B}\left(H_{x}\right): x \in X\right\}$ will denote a family of UCP maps defined on a separable operator system $S$. We introduce the bundle of dilations of the family $\phi$. Given a probability measure $\mu$ on $X$, we show that nontrivial Borelmeasurable sections of the dilation bundle exist whenever $\phi_{x}$ fails to be maximal for $\mu$-almost every $x \in X$.

Definition 5.1. By a dilation of the family $\phi$ we mean a Borel family of UCP maps $\psi=\left\{\psi_{x}: S \rightarrow \mathcal{B}\left(H_{x} \oplus \ell^{2}\right): x \in X\right\}$ that compresses pointwise to $\phi$ in the sense that

$$
\left\langle\psi_{x}(s) \xi, \eta\right\rangle=\left\langle\phi_{x}(s) \xi, \eta\right\rangle, \quad s \in S, \quad \xi, \eta \in H_{x}, \quad x \in X .
$$

Notice that the dilations of $\phi$ act on a Hilbert bundle $\left\{K_{x}: x \in X\right\}$ that is related to the Hilbert bundle $\left\{H_{x}: x \in X\right\}$ of $\phi$ in a particularly concrete way, namely $K_{x}=H_{x} \oplus \ell^{2}$ for all $x \in X$.

The dilations of $\phi$ are the Borel sections of a bundle $\mathcal{D}=\mathcal{D}^{\phi}$ that we now define as follows. For each $x \in X$, let $\mathcal{D}_{x}$ be the set of all UCP maps $\psi: S \rightarrow \mathcal{B}\left(H_{x} \oplus \ell^{2}\right)$ that compress to $\phi$ on $H_{x}$ :

$$
\langle\psi(s) \xi, \eta\rangle=\left\langle\phi_{x}(s) \xi, \eta\right\rangle, \quad s \in S, \quad \xi, \eta \in H_{x}
$$

The dilation bundle $p: \mathcal{D} \rightarrow X$ is the total space

$$
\mathcal{D}=\left\{(x, \psi): x \in X, \quad \psi \in \mathcal{D}_{x}\right\}
$$

with natural projection $p(x, \psi)=x$. We can view $\mathcal{D}$ as a subset of the bundle of maps $\operatorname{UCP}\left(X, S, \mathcal{B}\left(H \oplus \ell^{2}\right)\right)$, and as such it inherits a relative Borel structure making $p: \mathcal{D} \rightarrow X$ a Borel map.

Proposition 5.2. $\mathcal{D}$ is a Borel subset of $\operatorname{UCP}\left(X, S, \mathcal{B}\left(H \oplus \ell^{2}\right)\right)$, hence it is a standard Borel space. The dilations of $\phi$ are the Borel sections of the bundle $p$ : $\mathcal{D} \rightarrow X$.

Proof. For each $x \in X$, let $P_{x}$ be the projection of $H_{x} \oplus \ell^{2}$ onto $H_{x}$. For each $x \in X$ there is a natural compression map $\gamma_{x}$ of $\operatorname{UCP}\left(S, \mathcal{B}\left(H_{x} \oplus \ell^{2}\right)\right)$ onto $\operatorname{UCP}\left(S, \mathcal{B}\left(H_{x}\right)\right)$ defined by

$$
\gamma_{x}(\psi)(s)=P_{H_{x}} \psi(s) \uparrow_{H_{x}}, \quad s \in S,
$$

and the total map $\gamma$ is defined at the level of bundles by

$$
\gamma:(x, \psi) \in U C P\left(X, S, \mathcal{B}\left(H \oplus \ell^{2}\right)\right) \mapsto\left(x, \gamma_{x}(\psi)\right) \in U C P(X, S, \mathcal{B}(H)) .
$$

The total map $\gamma$ is a Borel map because both the inclusion $H_{x} \subseteq H_{x} \oplus \ell^{2}$ and its adjoint $P_{H_{x}}: H_{x} \oplus \ell^{2} \rightarrow H_{x}$ define Borel maps of (standard) Hilbert bundles whose fibers are, respectively, isometries and co-isometries. We can now exhibit $\mathcal{D}$ as a Borel set in $U C P\left(X, S, \mathcal{B}\left(H \oplus \ell^{2}\right)\right)$ by way of

$$
\mathcal{D}=\left\{(x, \psi) \in U C P\left(X, S, \mathcal{B}\left(H \oplus \ell^{2}\right)\right): \gamma(x, \psi)=\left(x, \phi_{x}\right)\right\},
$$

after noting that the graph $G=\left\{\left(x, \phi_{x}\right): x \in X\right\}$ of $x \mapsto \phi_{x}$ is a Borel subset of $\operatorname{UCP}(X, S, \mathcal{B}(H))$ (see Arv98, Theorem 3.3.2), and $\mathcal{D}=\gamma^{-1}(G)$.

The second sentence now follows from Remark 4.2, which characterizes Borel sections of bundles of UCP maps. 
In particular, note that for fixed $x \in X$, the fiber $\mathcal{D}_{x}=p^{-1}(x)$ is a closed convex subset of the BW-compact space of maps $\operatorname{UCP}\left(S, \mathcal{B}\left(H_{x} \oplus \ell^{2}\right)\right)$.

Proposition 5.3. Let $s \in S$, let $\xi$ be a Borel section of $\left\{H_{x}: x \in X\right\}$, and fix $x \in X$. Then $\phi_{x}$ is maximal on $(s, \xi(x))$ iff

$$
\|\psi(s) \xi(x)\| \leq\left\|\phi_{x}(s) \xi(x)\right\|, \quad \forall \psi \in \mathcal{D}_{x} .
$$

Proof. If $\phi_{x}$ is maximal on $(s, \xi(x))$, then for every dilation $\psi \in \mathcal{D}_{x}$ we have $\psi(s) \xi(x)=\phi_{x}(s) \xi(x)$, and (5.1) follows with equality. Conversely, assume that every dilation $\psi \in \mathcal{D}_{x}$ satisfies (5.1), and let $\omega: S \rightarrow \mathcal{B}(K)$ be an arbitrary dilation of $\phi_{x}$, with $K \supseteq H_{x}$. By the remarks following Definition 2.2, $\omega$ decomposes into a direct sum $\omega_{0} \oplus \lambda$ where $\omega_{0}$ acts on a separable Hilbert space $K_{0}$ with $K \supseteq K_{0} \supseteq H_{x}$. We can identity $K_{0}$ with either $H_{x} \oplus \mathbb{C}^{k}$ or $H_{x} \oplus \ell^{2}$ and replace $\omega_{0}$ with a unitarily equivalent map $\omega_{0}^{\prime}$ from $S$ to the respective space of operators such that $\phi_{x} \preceq \omega_{0}^{\prime}$. If $K_{0}=H_{x} \oplus \ell^{2}$, then we set $\psi=\omega_{0}^{\prime}$; otherwise we set $\psi=\omega_{0}^{\prime} \oplus \mu: S \rightarrow \mathcal{B}\left(H_{x} \oplus \ell^{2}\right)$ where $\mu$ is an arbitrary UCP map from $S$ into $\mathcal{B}\left(\ell^{2} \ominus \mathbb{C}^{k}\right)$ that fills out the difference. Thus in all cases we have exhibited a map $\psi \in \mathcal{D}_{x}$ such that $\|\omega(s) \xi(x)\| \leq\|\psi(s) \xi(x)\|$. From (5.1) it follows that

$$
\begin{aligned}
\left\|\omega(s) \xi(x)-\phi_{x}(s) \xi(x)\right\|^{2} & =\left\|\omega(s) \xi(x)-P_{H_{x}} \omega(s) \xi(x)\right\|^{2} \\
& =\|\omega(s) \xi(x)\|^{2}-\left\|P_{H_{x}} \omega(s) \xi(x)\right\|^{2} \\
& \leq\|\psi(s) \xi(x)\|^{2}-\left\|\phi_{x}(s) \xi(x)\right\|^{2} \leq 0 .
\end{aligned}
$$

Hence $\omega(s) \xi(x)=\phi_{x}(s) \xi(x)$, so that $\phi_{x}$ is maximal on $(s, \xi(x))$.

Definition 5.4. Fix a probability measure $\mu$ on $X$. A family of UCP maps $\phi=$ $\left\{\phi_{x}: S \rightarrow \mathcal{B}\left(H_{x}\right)\right\}$ is said to be maximal $\mu$-almost everywhere if there is a Borel set $N \subseteq X$ such that $\mu(N)=0$ and $\phi_{x}$ is maximal for all $x \in X \backslash N$.

Lemma 5.5. Let $\phi=\left\{\phi_{x}: S \rightarrow \mathcal{B}\left(H_{x}\right): x \in X\right\}$ be a family of UCP maps of $S$ and let $\mu$ be a probability measure on $X$. If for every $s \in S$ and every Borel section $\xi: x \in X \rightarrow H_{x}$ of $\left\{H_{x}: x \in X\right\}$ there is a Borel set $N_{s, \xi} \subseteq X$ such that $\mu\left(N_{s, \xi}\right)=0$ and $\phi_{x}$ satisfies (5.1) for every $x \in X \backslash N_{s, \xi}$, then $\phi$ is maximal $\mu$-almost everywhere.

Proof. Let $C$ be a countable subset of $S$ that is dense in $S$ and let $D=\left\{\xi_{1}, \xi_{2}, \ldots\right\}$ be a sequence of Borel sections of the Hilbert bundle $\left\{H_{x}: x \in X\right\}$ such that $\left\{\xi_{1}(x), \xi_{2}(x), \ldots\right\}$ is dense in $H_{x}$ for every $x \in X$. By hypothesis, for every $(s, \xi) \in$ $C \times D$, there is a Borel set $N_{s, \xi}$ of measure zero such that

$$
\sup \left\{\|\psi(s) \xi(x)\|: \psi \in \mathcal{D}_{x}\right\} \leq\left\|\phi_{x}(s) \xi(x)\right\|, \quad x \in X \backslash N_{s, \xi} .
$$

Let $N$ be the (countable) union of the sets $N_{s, \xi},(s, \xi) \in C \times D$. Then $\mu(N)=0$, and for every $x \in X \backslash N$ and every $\psi \in \mathcal{D}_{x}$, we have

$$
\|\psi(s) \xi(x)\| \leq\left\|\phi_{x}(s) \xi(x)\right\|, \quad s \in C, \quad \xi \in D .
$$

It follows that when $x \in X \backslash N$ we have $\|\psi(s) \eta\| \leq\left\|\phi_{x}(s) \eta\right\|$ for every $s \in S=\bar{C}$, every $\eta \in H_{x}=\overline{\left\{\xi_{1}(x), \xi_{2}(x), \ldots\right\}}$, and every $\psi \in \mathcal{D}_{x}$. Proposition 5.3 implies that $\phi_{x}$ is maximal for every $x \in X \backslash N$.

The following result provides the key step in the proof of Theorem 6.1 
Theorem 5.6. Let $\mu$ be a probability measure on $X$ and let

$$
\phi=\left\{\phi_{x}: S \rightarrow \mathcal{B}\left(H_{x}\right): x \in X\right\}
$$

be a family of UCP maps that fails to be maximal $\mu$-almost everywhere. Then there is an operator $s \in S$, a Borel section $\xi$ of $\left\{H_{x}: x \in X\right\}$, a Borel subset $X_{0} \subseteq X$ of positive measure and a Borel function $x \in X_{0} \mapsto \psi_{x} \in \mathcal{D}_{x}$ such that

$$
\left\|\psi_{x}(s) \xi(x)\right\|>\left\|\phi_{x}(s) \xi(x)\right\|, \quad x \in X_{0} .
$$

In particular, for each $x \in X_{0}, \psi_{x}$ is a dilation of $\phi_{x}$ that does not decompose into a direct sum $\phi_{x} \oplus \lambda_{x}$.

Proof. By Lemma 5.5 there is an operator $s \in S$ and a Borel section $\xi: x \in X \mapsto$ $\xi(x) \in H_{x}$ with the property that $\phi_{x}$ fails to satisfy (5.1) $\mu$-almost everywhere. This simply means that if $E \subseteq X$ is a Borel set with the property that

$$
\sup _{\psi \in \mathcal{D}_{x}}\|\psi(s) \xi(x)\| \leq\left\|\phi_{x}(s) \xi(x)\right\|
$$

for every $x \in E$, then $X \backslash E$ must have positive measure.

Let $\eta_{1}, \eta_{2}, \ldots$ be a sequence of Borel sections of $\left\{H_{x}: x \in X\right\}$ such that $\left\{\eta_{1}(x), \eta_{2}(x), \ldots\right\}$ is dense in the unit ball of $H_{x}$ for all $x \in X$, and consider the sequence of functions $F_{k}: \mathcal{D} \rightarrow[0, \infty), k \geq 1$, defined by

$$
F_{k}(x, \psi)=\left|\left\langle\psi(s) \xi(x), \eta_{k}(x)\right\rangle\right|, \quad \psi \in \mathcal{D}_{x}, \quad x \in X .
$$

The $F_{k}$ are Borel functions, they restrict to continuous functions on each fiber $\mathcal{D}_{x}$, and they have the property that $\phi_{x}$ is maximal on $(s, \xi(x))$ iff

$$
F_{k}(x, \psi) \leq\left\|\phi_{x}(s) \xi(x)\right\|, \quad k=1,2, \ldots, \quad \psi \in \mathcal{D}_{x} .
$$

Consider the following subset of $\mathcal{D}$ :

$$
\mathcal{D}_{+}=\bigcup_{k=1}^{\infty}\left\{(x, \psi) \in \mathcal{D}: F_{k}(x, \psi)>\left\|\phi_{x}(s) \xi(x)\right\|\right\} .
$$

Being exhibited as a countable union of Borel sets, $\mathcal{D}_{+}$is a Borel subset of $\mathcal{D}$, and the natural projection $p: \mathcal{D} \rightarrow X$ restricts to a Borel map of $\mathcal{D}_{+}$into $X$. Let $X_{+}=p\left(\mathcal{D}_{+}\right)$be the range of the restricted map. While $X_{+}$is not necessarily a Borel set, Theorem 3.3 implies that it is an analytic subset of $X$ and is therefore absolutely measurable. Notice too that, by its definition, $X_{+}$is the set of all $x \in X$ such that the UCP map $\phi_{x}: S \rightarrow \mathcal{B}\left(H_{x}\right)$ is not maximal on $(s, \xi(x))$.

Since $X_{+}$is absolutely measurable, there exist Borel sets $E_{\mu} \subseteq X_{+} \subseteq F_{\mu}$ such that $\mu\left(F_{\mu} \backslash E_{\mu}\right)=0$. Note that $\phi_{x}$ is maximal at $(s, \xi(x))$ for every $x \in X \backslash F_{\mu} \subseteq$ $X \backslash X_{+}$. So by our choice of $s$ and $\xi, F_{\mu}$ must have positive measure. Hence $E_{\mu}$ is a Borel subset of $X_{+}$of the same positive measure, and we may consider the restricted map $p: \mathcal{D}_{0} \rightarrow E_{\mu}$ defined by

$$
\mathcal{D}_{0}=\left\{(x, \psi) \in \mathcal{D}_{+}: x \in E_{\mu}\right\}, \quad p(x, \psi)=x .
$$

The projection $p: \mathcal{D}_{0} \rightarrow E_{\mu}$ is a surjective Borel function and both $\mathcal{D}_{0}$ and $E_{\mu}$ are standard Borel spaces. By Theorem 3.2 there is a Borel set $N \subseteq E_{\mu}$ of $\mu$-measure zero and a Borel cross section $x \in E_{\mu} \backslash N \mapsto \psi_{x} \in \mathcal{D}_{x}$ for $p$. This function $x \mapsto \psi_{x}$ satisfies (5.2) for $X_{0}=E_{\mu} \backslash N$. 


\section{Structure of maps with the unique extension property}

Given a UCP map $\phi: S \rightarrow \mathcal{B}(H)$ with the unique extension property, it is easy to show that for every decomposition $\phi=\phi_{1} \oplus \phi_{2}$ of $\phi$ into a direct sum of UCP maps of $S$, both $\phi_{1}$ and $\phi_{2}$ inherit the unique extension property. The purpose of this section is to generalize that fact to infinite continuous decompositions of $\phi$ into a direct integral. Throughout this section, $(X, \mu)$ will denote a standard Borel probability space.

Theorem 6.1. Let $\left\{H_{x}: x \in X\right\}$ be a standard Borel Hilbert bundle over $X$, let $\phi=\left\{\phi_{x}: S \rightarrow \mathcal{B}\left(H_{x}\right): x \in X\right\}$ be a family of UCP maps, and let $\mu$ be a probability measure on $X$. Let

$$
H=\int_{X}^{\oplus} H_{x} d \mu(x)
$$

be the direct integral of Hilbert spaces and let $\phi: S \rightarrow \mathcal{B}(H)$ be the direct integral of UCP maps

$$
\phi(s)=\int_{X}^{\oplus} \phi_{x}(s) d \mu(x), \quad s \in S .
$$

If $\phi$ has the unique extension property, then there is a Borel set $N \subseteq X$ of measure zero such that $\phi_{x}: S \rightarrow \mathcal{B}\left(H_{x}\right)$ has the unique extension property for every $x$ in $X \backslash N$.

Proof. Contrapositively, assume that the direct integral $\phi$ has the unique extension property but that there is no Borel set $N$ with $\mu(N)=0$ such that $\phi=\left\{\phi_{x}: S \rightarrow\right.$ $\left.\mathcal{B}\left(H_{x}\right)\right\}$ has the unique extension property for every $x \in X \backslash N$.

By Theorem 5.6, there is a Borel set $X_{0} \subseteq X$ of positive measure and a Borel section $\psi: x \in X_{0} \rightarrow \psi_{x} \in U C P\left(S, \mathcal{B}\left(H_{x} \oplus \ell^{2}\right)\right)$ that dilates the restricted family $\left\{\phi_{x}: S \rightarrow \mathcal{B}\left(H_{x}\right): x \in X_{0}\right\}$ nontrivially for every $x \in X_{0}$. Thus, for every $x \in X_{0}$, $\psi_{x}$ is a dilation of $\phi_{x}$ that cannot be decomposed into a direct sum $\phi_{x} \oplus \lambda_{x}$. Define a larger bundle of separable Hilbert spaces $\left\{K_{x}: x \in X\right\}$ by

$$
K_{x}= \begin{cases}H_{x} \oplus \ell^{2}, & x \in X_{0}, \\ H_{x}, & x \in X \backslash X_{0},\end{cases}
$$

and a new measurable family $\tilde{\phi}=\left\{\tilde{\phi}_{x}: S \rightarrow \mathcal{B}\left(K_{x}\right): x \in X\right\}$ by

$$
\tilde{\phi}_{x}= \begin{cases}\psi_{x}, & x \in X_{0} \\ \phi_{x}, & x \in X \backslash X_{0}\end{cases}
$$

The family $\left\{\tilde{\phi}_{x}: S \rightarrow \mathcal{B}\left(K_{x}\right): x \in X\right\}$ is a dilation of the original family $\left\{\phi_{x}: S \rightarrow\right.$ $\left.\mathcal{B}\left(H_{x}\right): x \in X\right\}$, and we can form the direct integral of UCP maps of $S$

$$
\tilde{\phi}=\int_{X}^{\oplus} \tilde{\phi}_{x} d \mu(x)
$$

acting on the Hilbert space

$$
K=\int_{X}^{\oplus} K_{x} d \mu(x) .
$$

For each $x \in X$, let $P_{x}$ be the projection of $K_{x}$ on $H_{x}$, so that the projection $P$ of $K$ on $H$ decomposes into a direct integral

$$
P=\int_{X}^{\oplus} P_{x} d \mu(x)
$$


The UCP map $\tilde{\phi}$ is a dilation of $\phi$, and $\phi$ is maximal since it has the unique extension property. Hence $P$ commutes with $\tilde{\phi}(S)$. Let $\left\{a_{1}, a_{2}, \ldots\right\}$ be a countable norm-dense subset of $S$. Since $P$ is a decomposable operator that commutes with the sequence of decomposable operators $\left\{\tilde{\phi}\left(a_{1}\right), \tilde{\phi}\left(a_{2}\right), \ldots\right\}$, it follows that $P_{x}$ must commute with $\left\{\tilde{\phi}_{x}\left(a_{1}\right), \tilde{\phi}_{x}\left(a_{2}\right), \ldots\right\}$ for all $x$ in the complement of some $\mu$-null Borel set $N \subseteq X$, and hence $P_{x}$ commutes with $\tilde{\phi}_{x}(S)$ for all $x \in X \backslash N$. In particular, for $x \in X_{0} \backslash N$, this implies that the constructed family of dilations $\psi_{x}$ decomposes into a direct sum of UCP maps

$$
\psi_{x}=\phi_{x} \oplus \lambda_{x}
$$

contradicting the stated property of $\psi$ on a set of positive measure.

\section{EXISTENCE OF BOUNDARY REPRESENTATIONS}

In this section we prove the following main result.

Theorem 7.1. Every separable operator system $S \subseteq C^{*}(S)$ has sufficiently many boundary representations.

Proof. We will show that there is a set $\left\{\sigma_{x}: x \in A\right\}$ of boundary representations that satisfies (1.1). To that end, we realize $S \subseteq \mathcal{B}\left(H_{0}\right)$ as an operator system acting on a separable Hilbert space $H_{0}$. By Theorem 2.5, there is a separable Hilbert space $H \supseteq H_{0}$ and a UCP map $\phi: S \rightarrow \mathcal{B}(H)$ with the unique extension property such that $P_{H_{0}} \phi(s) \uparrow_{H_{0}}=s, s \in S$. Obviously, $\phi$ is a complete isomorphism of $S$ onto $\phi(S) \subseteq \mathcal{B}(H)$.

Choose a maximal abelian von Neumann subalgebra $\mathcal{M}$ of the commutant $\phi(S)^{\prime}$. Since $\mathcal{M}$ acts on a separable Hilbert space its unit ball is weak*-compact metrizable, hence $\mathcal{M}$ contains a separable unital $C^{*}$-algebra $\mathcal{A}$ whose weak ${ }^{*}$ closure is $\mathcal{M}$. The Gelfand spectrum $X$ of $\mathcal{A}$ is a compact metrizable space, hence we may view it as a standard Borel space, and there is a probability measure $\mu$ on $X$ such that $\mathcal{M} \cong L^{\infty}(X, \mu)$.

Conventional multiplicity theory implies that there is a standard Hilbert bundle $\left\{H_{x}: x \in X\right\}$ that gives rise to a decomposition

$$
H=\int_{X}^{\oplus} H_{x} d \mu(x)
$$

in such a way that $\mathcal{M}$ is realized as the algebra of multiplications by scalar functions in $L^{\infty}(X, \mu)$. For example, this encapsulates the discussion on page 55 of Arv98. In these "coordinates", $\phi$ becomes a UCP map of $S$ into $\mathcal{B}(H)$ whose range $\phi(S) \subseteq \mathcal{M}^{\prime}$ consists of decomposable operators. The $C^{*}$-algebra generated by $\phi(S)$ is separable, hence the $C^{*}$-algebra $\mathcal{B}$ generated by $\mathcal{A} \cup \phi(S)$ is a separable $C^{*}$-algebra contained in the commutant of $\mathcal{M}$. Note that by the choice of $\mathcal{A}$, the commutant of $\mathcal{B}$ is $\mathcal{M}$, so by the double commutant theorem, $\mathcal{B}$ is weak ${ }^{*}$-dense in the von Neumann algebra $\mathcal{M}^{\prime}$ of all decomposable operators.

Corollary 2 of Theorem 4.2.1 of Arv98, together with the "flattening and piecing together" procedure described in Section 4 implies that there is a Borel-measurable family of representations $\pi_{x}: \mathcal{B} \rightarrow \mathcal{B}\left(H_{x}\right), x \in X$, with the property

$$
b=\int_{X}^{\oplus} \pi_{x}(b) d \mu(x), \quad b \in \mathcal{B} .
$$


Since $\mathcal{B}$ is weak*- dense in $\mathcal{M}^{\prime}$, Corollary 2 of Proposition 4.2 .2 of Arv98 implies that $\pi_{x}(\mathcal{B})$ is an irreducible set of operators for almost every $x \in X$. By discarding a Borel set of measure zero from $X$, we can assume that $\pi_{x}(\mathcal{B})$ is an irreducible $C^{*}$ algebra for every $x \in X$. Finally, since $\mathcal{A}$ consists of scalar multiplication operators, we have $\pi_{x}(\mathcal{A})=\mathbb{C} \cdot \mathbf{1}_{H_{x}}$ for all $x \in X$.

We can now define a family of UCP maps $\left\{\phi_{x}: S \rightarrow \mathcal{B}\left(H_{x}\right): x \in X\right\}$ by setting $\phi_{x}(a)=\pi_{x}(\phi(a)), a \in S, x \in X$, thereby obtaining a disintegration of $\phi$ into a direct integral of UCP maps

$$
\phi(a)=\int_{X}^{\oplus} \phi_{x}(a) d \mu(x), \quad a \in S .
$$

After noting that for every $x \in X$,

$$
\pi_{x}(\mathcal{B})=\pi_{x}\left(C^{*}(\phi(S) \cup \mathcal{A})\right)=C^{*}\left(\pi_{x}(\phi(S) \cup \mathcal{A})\right)=C^{*}\left(\phi_{x}(S)\right),
$$

one finds that $\phi_{x}(S)$ is an irreducible operator system for every $x \in X$. Thus, $\left\{\phi_{x}: S \rightarrow \mathcal{B}\left(H_{x}\right): x \in X\right\}$ defines a (Borel) family of irreducible UCP maps of $S$. The decomposition (7.1) itself implies that

$$
\|a\|=\|\phi(a)\|=\operatorname{ess} \sup _{x \in X}\left\|\phi_{x}(a)\right\|, \quad a \in S,
$$

ess sup denoting the essential supremum with respect to the measure $\mu$. Note that similar formulas hold throughout the matrix hierarchy over $S$. Indeed, for each $n=2,3, \ldots$, the direct sum $n \cdot H$ of $n$ copies of $H$ decomposes into a direct integral

$$
n \cdot H=\int_{X}^{\oplus} n \cdot H_{x} d \mu(x),
$$

and the associated map of $n \times n$ matrices $\left(a_{i j}\right) \in M_{n}(S) \mapsto\left(\phi\left(a_{i j}\right)\right) \in \mathcal{B}(n \cdot H)$ admits a similar direct integral decomposition in which the $n \times n$ operator matrix $\left(\phi\left(a_{i j}\right)\right)$ is realized as a direct integral of $n \times n$ operator matrices over $\mathcal{B}\left(H_{x}\right), x \in X$,

$$
\left(\phi\left(a_{i j}\right)\right)=\int_{X}^{\oplus}\left(\phi_{x}\left(a_{i j}\right)\right) d \mu(x) .
$$

From this formula one concludes that for every $n=2,3, \ldots$,

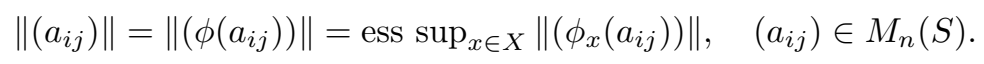

By Theorem 6.1, there is a Borel set $N \subseteq X$ of measure zero such that for each $x \in X \backslash N, \phi_{x}$ has the unique extension property, and therefore defines an element $\sigma_{x}$ of $\partial_{S}$. Since $S$ is separable, we can restrict all terms $a_{i j}$ appearing in the formulas of (7.2) to a countable dense subset $C$ of $S$, thereby obtaining an equivalent countable set of formulas. Finally, since the essential supremum of an $L^{\infty}$ function defined on a measure space is the pointwise supremum of that function restricted to a subset whose complement is of measure zero, there is a single Borel set $A \subseteq X$, such that $\mu(X \backslash A)=0$, with the property that the entire sequence of formulas (7.2) holds as pointwise suprema - over $x \in A$ - of norms of matrix functions whose entries $\phi_{x}\left(a_{i j}\right)$ involve terms with $a_{i j} \in C$. Finally, since $C$ is dense in $S$, it follows that the set of boundary representations $\left\{\sigma_{x}: x \in A\right\}$ satisfies (1.1). 


\section{Pure states of $S$}

In this section we sharpen Theorem 7.1 by showing that every pure state of $S$ can be associated with a boundary representation.

Definition 8.1. A state $\rho$ of $C^{*}(S)$ is called an $S$-boundary state if it is a pure state of $C^{*}(S)$ and the irreducible representation $\pi$ occurring in its GNS representation

$$
\rho(x)=\langle\pi(x) \xi, \xi\rangle, \quad x \in C^{*}(S),
$$

is a boundary representation for $S$.

By a state of $S$ we mean a positive linear functional $\rho$ on $S$ satisfying $\rho(\mathbf{1})=1$. A pure state of $S$ is an extreme point of the convex weak*-compact set of all states of $S$.

Theorem 8.2. Every pure state of a separable operator system $S$ can be extended to an $S$-boundary state of $C^{*}(S)$.

The assertion is that every pure state $\rho$ of $S$ can be written the form (8.1) $\rho(a)=\langle\pi(a) \xi, \xi\rangle, a \in S$, for some $\pi \in \partial_{S}$. It is not hard to apply Theorem 8.2 judiciously throughout the matrix hierarchy $M_{n}(S), n=1,2, \ldots$, to deduce that $S$ has sufficiently many boundary representations; and in that sense Theorem 8.2 contains Theorem 7.1 (we omit those details). The proof of Theorem 8.2 requires the following measure-theoretic refinement of the notion of extreme point:

Lemma 8.3. Let $\phi$ be a pure state of $S$ and let $(X, \mu)$ be a standard Borel probability space. For every $x \in X$, let $\rho_{x}$ be a state of $S$ such that for every $a \in S, \rho_{x}(a)$ is a Borel-measurable function of $x$, satisfying

$$
\phi(a)=\int_{X} \rho_{x}(a) d \mu(x), \quad a \in S .
$$

Then $N=\left\{x \in X: \rho_{x} \neq \phi\right\}$ is a Borel set of measure zero.

Proof. Since $S$ is separable, its state space $Y$ is a compact convex metrizable space relative to its weak* topology, and the measurability hypothesis on the function $\rho$ implies that $x \in X \mapsto \rho_{x} \in Y$ is a Borel map of $X$ into $Y$. Let $\nu$ be the push-forward of $\mu$, i.e., the probability measure on $Y$ defined on Borel sets by

$$
\nu(E)=\mu\left\{x \in X: \rho_{x} \in E\right\}, \quad E \subseteq Y .
$$

By the standard change-of-variables formula of measure theory, for every bounded Borel function $F: Y \rightarrow \mathbb{C}$ we have

$$
\int_{X} F\left(\rho_{x}\right) d \mu(x)=\int_{Y} F(\rho) d \nu(\rho) .
$$

Taking $F(\rho)=\rho(a)$ for fixed $a \in S$, we obtain

$$
\int_{X} \rho_{x}(a) d \mu(x)=\int_{Y} \rho(a) d \nu(\rho),
$$

and hence

$$
\phi(a)=\int_{Y} \rho(a) d \nu(\rho), \quad a \in S .
$$

A result of Bauer (see Proposition 1.4 of [Phe66] or [Phe01]) implies that $\nu$ is the point mass concentrated at $\phi$. Let $N=\left\{x \in X: \rho_{x} \neq \phi\right\}$. Then $N$ is a Borel subset of $X$ such that $\mu(N)=\nu(Y \backslash\{\phi\})=0$. 
Proof of Theorem 8.2. Let $\rho$ be an extension of $\phi$ to a state of $C^{*}(S)$, and let $\rho(x)=\left\langle\pi_{0}(x) \xi, \xi\right\rangle, x \in C^{*}(S)$, be its GNS representation. Note that the Hilbert space $\left[\pi_{0}\left(C^{*}(S)\right) \xi\right]$ of $\pi_{0}$ is separable. By Theorem 2.5. the UCP map $\pi_{0}\left\lceil_{S}\right.$ can be dilated to a UCP map of $S$, on a larger separable Hilbert space, which has the unique extension property. Let $\pi$ be the extension of this dilation to $C^{*}(S)$. The formula $\phi(a)=\langle\pi(a) \xi, \xi\rangle$ persists for $a \in S$.

As in the proof of Theorem 7.1 we can decompose $\pi$ into a direct integral of irreducible representations $\pi_{x}: C^{*}(S) \rightarrow \mathcal{B}\left(H_{x}\right)$ parameterized by a standard Borel probability space $(X, \mu)$. By Theorem 6.1, $\pi_{x}$ is a boundary representation for almost every $x \in X$. The vector $\xi$ becomes a square-integrable section $x \in$ $X \mapsto \xi(x) \in H_{x}$, and we can define a Borel section of unit vectors over the Borel set $X_{0}=\{x \in X: \xi(x) \neq 0\}$ by

$$
e(x)=\|\xi(x)\|^{-1} \xi(x), \quad x \in X_{0} .
$$

This decomposition of $\pi$ leads to the following representation of $\phi$ :

$$
\begin{aligned}
\phi(a) & =\int_{X}\left\langle\pi_{x}(a) \xi(x), \xi(x)\right\rangle d \mu(x) \\
& =\int_{X_{0}}\left\langle\pi_{x}(a) e(x), e(x)\right\rangle\|\xi(x)\|^{2} d \mu(x)=\int_{X_{0}}\left\langle\pi_{x}(a) e(x), e(x)\right\rangle d \nu(x),
\end{aligned}
$$

for $a \in S$, where $\nu$ is the probability measure defined on $X_{0}$ by

$$
d \nu(x)=\|\xi(x)\|^{2} d \mu(x) .
$$

Note that $\rho_{x}(b)=\left\langle\pi_{x}(b) e(x), e(x)\right\rangle$ is an $S$-boundary state of $C^{*}(S)$ for every $x \in X_{0}$. Since $\phi$ is a pure state of $S$, Lemma 8.3 implies that

$$
\phi(a)=\rho_{x}(a)=\left\langle\pi_{x}(a) e(x), e(x)\right\rangle, \quad a \in S,
$$

for all $x$ in the complement $X_{0} \backslash N$ of a Borel set of $\nu$-measure zero, thereby exhibiting many $S$-boundary states that extend $\phi$.

\section{Concluding Remarks}

The proof of Theorem 7.1 is far from constructive. Rather, it is more akin to probabilistic arguments whereby one establishes the existence of a desired property by constructing a nonvacuous probability space in which the property can be shown to hold almost surely. The proof of Theorem 8.2 illustrates the technique in this context.

Naturally, it would be desirable to get rid of the disintegration theory that is seriously exploited above by finding a more direct construction of boundary representations. A preliminary attempt to do that was made in Arv69, but without much success. Indeed, it is still unclear how one might effectively characterize the pure states of $C^{*}(S)$ whose GNS representations are boundary representations for $S$. For example, can every pure state $\rho$ of $S$ be extended to a pure state $\tilde{\rho}$ of $C^{*}(S)$ whose GNS representation is a boundary representation for $S$ ? The answer is yes if $S$ is separable by Theorem 8.2, or if $C^{*}(S)$ is commutative in general. What we are proposing is a more direct proof that will work for inseparable operator systems. 
As a test problem for such developments, we propose:

Problem. Does Theorem 7.1 remain true for inseparable operator systems?

Perhaps it is worth pointing out that in general, heroic attempts to get rid of separability hypotheses for problems in operator algebras can force one to look carefully at the fundamentals of set theory. For example, Akemann and Weaver AW04 have constructed a counterexample to Naimark's problem by making use of a set-theoretic principle that is known to be consistent with, but not provable from, the standard axioms of set theory. They also showed that the statement There is a counterexample to Naimark's problem that is generated by $\aleph_{1}$ elements is undecidable within standard set theory.

\section{ACKNOWLEDGMENTS}

Finally, it is with pleasure that I acknowledge valuable conversations during the fall of 2002 with Narutaka Ozawa, who completely understood the first version of DM05 when I did not. Those conversations led to an unpublished exposition of the results of DM05 in Arv03. In particular, Lemma 2.7 was inspired by an observation of Ozawa. Without the paper DM05 or Ozawa's visit to Berkeley, this paper would most likely not exist. I would also like to thank the anonymous referee, whose careful reading of the manuscript led to many perceptive comments that significantly improved the readability of this paper.

\section{REFERENCES}

[Agl88] Jim Agler. An abstract approach to model theory. In Surveys of some recent results in operator theory, volume II, pages 1-23, Harlow, 1988. Longman Sci. Tech. MR976842 (90a:47016)

[Arv69] W. Arveson. Subalgebras of $C^{*}$-algebras. Acta Math., 123:141-224, 1969. MR0253059 $(40: 6274)$

[Arv72] W. Arveson. Subalgebras of $C^{*}$-algebras II. Acta Math., 128:271-308, 1972. MR.0394232 (52:15035)

[Arv98] W. Arveson. An invitation to $C^{*}$-algebras, volume 39 of Graduate Texts in Mathematics. Springer-Verlag, New York, reprinted 1998. MR0512360 (58:23621)

[Arv03] W. Arveson. Notes on the unique extension property. Unpublished, 2003. Available from http://math. berkeley.edu/ ${ }^{\sim}$ arveson/Dvi/unExt.pdf.

[AW04] C. Akemann and N. Weaver. Consistency of a counterexample to Naimark's problem. Proc. NAS (USA), 101:7522-7525, May 2004. MR2057719 (2004m:46131)

[Bla68] D. Blackwell. A Borel set not containing a graph. Ann. Math. Stat., 39(4):1345-1347, 1968. MR0229451 (37:5025)

[Ble01] D. Blecher. The Shilov boundary of an operator space and the characterization theorems. J. Funct. Anal., 182:280-343, 2001. MR.1828796 (2002d:46049)

[BLM04] D. Blecher and C. Le Merdy. Operator algebras and their modules, volume 30 of LMS Monographs. Clarendon Press, Oxford, 2004. MR2111973 (2006a:46070)

[Dix57] J. Dixmier. Les algèbres d'opèrateurs dans l'espace Hilbertien. Gauthier-Villars, Paris, 1957. MR0094722 (20:1234)

[DM05] M. Dritschel and S. McCullough. Boundary representations for families of representations of operator algebras and spaces. J. Operator Theory, 53(1):159-167, 2005. MR 2132691 (2006a:47095)

[Ham79a] M. Hamana. Injective envelopes of $C^{*}$-algebras. J. Math. Soc. Japan, 31:181-197, 1979. MR519044 (80g:46048)

[Ham79b] M. Hamana. Injective envelopes of operator systems. Publ. Res. Inst. Math. Sci. (Kyoto), 15:773-785, 1979. MR566081 (81h:46071)

[Lus30] N. Lusin. Sur le probleme de M. J. Hadamard d'uniformisation des ensembles. Comtes Rendus Acad. Sci. Paris, 190:349-351, 1930. 
[MS98] P. Muhly and B. Solel. An algebraic characterization of boundary representations. In Nonselfadjoint operator algebras, operator theory, and related topics, pages 189-196, Basel, 1998. Birkhäuser. MR1639657 (99f:47057)

[Nov31] P. Novikoff. Sur les fonctions implicites measurables B. Fund. Math., 17:8-25, 1931.

[Pau02] V. Paulsen. Completely bounded maps and operator algebras. Cambridge University Press, Cambridge, UK, 2002. MR1976867 (2004c:46118)

[Phe66] R. R. Phelps. Lectures on Choquet's theorem, volume 7 of Van Nostrand Mathematical Studies. Van Nostrand, Princeton, 1966. MR0193470 (33:1690)

[Phe01] R. R. Phelps. Lectures on Choquet's theorem, volume 1757 of Lecture Notes in Mathematics. Springer-Verlag, Berlin, second edition, 2001. MR.1835574 (2002k:46001)

Department of Mathematics, University of California, Berkeley, California 94720

E-mail address: arveson@math.berkeley.edu 